\title{
La investigación ante los desafíos de los escenarios de aprendizaje futuros
}

\author{
Research in the Face of the Challenges of Future Learning Scenarios \\ Jesús Salinas \\ Universitat de les Illes Balears, Palma de Mallorca, España \\ jesus.salinas@uib.es
}

\begin{abstract}
Resumen
La investigación sobre escenarios de aprendizaje futuros se orienta a la adecuada integración de las posibilidades pedagógicas de los entornos personales, sociales e institucionales (LMS) de aprendizaje, y en concreto, a definir metodologías de enseñanzaaprendizaje (e-a) para estos nuevos entornos virtuales integrados más abiertos e innovadores.

El planteamiento es que las instituciones de educación superior no pueden explotar los entornos virtuales de enseñanza-aprendizaje en el futuro próximo apoyándose solamente en los entornos institucionalizados (LMS al uso), sino que requerirán unos entornos abiertos, flexibles, innovadores, que integren los entornos personales, sociales e institucionales. Para ello, no solo se ha de modificar el enfoque educativo utilizado en las experiencias de entornos virtuales, se ha de considerar la importancia de los sistemas de gestión personal e institucional de la información, el control del proceso de aprendizaje por parte del usuario, los espacios compartidos, la organización del aprendizaje colaborativo, la incorporación de metodologías centradas en el alumnos.

Para comprender mejor la investigación sobre escenarios de aprendizaje y sus implicaciones, ésta es situada en el contexto de la agenda de investigación actual relacionada con la Tecnología Educativa.
\end{abstract}

Palabras clave: Escenario de aprendizaje, entorno virtual, entorno personal de aprendizaje, PLE.

\begin{abstract}
The purpose of research on future learning scenarios aims at proper exploitation of the pedagogical possibilities of the personal, social and institutional learning environments (LMS), especially to define teaching and learning methodologies for these new integrated virtual environments more, open and innovative.

The approach is that institutions of higher education cannot exploit the teaching and learning virtual environments in the near future relying solely in the institutional environments. They will require open, flexible and innovative environments to integrate the personal, social and institutional environments. For this purpose, not only the educational approach used should be modified regarding virtual environments experiences, but the importance of personal and institutional management systems, the teaching process control by the user, the shared spaces, the collaborative learning organization and the incorporation of methodologies centered in the student, have all to be considered.

To better understand the research on learning scenarios and their implications, this research is situated in the context of the current research agenda related to Educational Technology.
\end{abstract}

Keywords: Learning scenario, virtual environment, personal learning environment, PLE. 


\section{Los escenarios de aprendizaje en la agenda de investigación educativa}

Si entendemos la universidad del futuro como una institución que suministra formación a una mayoría de la población a lo largo de toda la vida, como una institución generadora de conocimiento, aunque no la única, y como una organización al servicio de las necesidades de formación y de desarrollo tecnológico del entorno, dentro de un modelo de sociedad que ha sido definido como la sociedad del aprendizaje o del conocimiento, los escenarios que en ella se configuran para albergar procesos de enseñanza-aprendizaje se constituyen elementos privilegiados de pensamiento pedagógico.

La investigación sobe escenarios de aprendizaje podemos situarla en la zona de tensión entre la tradición didáctica, de donde podemos lograr sus fundamentos, y la necesidad de adaptarse a la actualidad, lo que supone incorporar cambios metodológicos, en algunos casos, condicionados por las características tecnológicas de los entornos de comunicación donde se desarrolla el proceso didáctico. Las instituciones de educación superior tienen que adaptarse a nuevas modalidades de formación más acordes con las necesidades que esta nueva sociedad presenta: desde aulas convencionales unidas a través de la red a grupos de trabajo colaborativo en contextos totalmente a distancia, desde clases de la educación formal a comunidades de práctica o al aprendizaje incidental. De ahí lo necesario de ocuparse y de reflexionar sobre ello si pretendemos abordar con sensatez, desde la óptica pedagógica, las perspectivas de futuro que la evolución de las TIC van ofreciendo.

\section{La investigación sobre escenarios de aprendizaje}

Ocuparse de los escenarios de aprendizaje tiene importantes implicaciones respecto a la investigación en dos líneas que, desde nuestra perspectiva, no pueden investigarse por separado (Salinas, 2009a): ¿cuáles son y cómo se configuran los escenarios de aprendizaje futuros? Y ¿Cómo van a tener que desenvolverse los actores del proceso de enseñanza-aprendizaje?

Numerosos autores se han ocupado de estas cuestiones: Paulsen (2004), Koper (2004), Mason y Rennjie (2008) o Conole (2008) ofrecen avances de cara a elaborar modelos educativos para elearning. De Benito (2006), Weller (2007) entre otros muchos, estudian las posibilidades que las aplicaciones de gestión y distribución de materiales en la web ofrecen desde la perspectiva pedagógica. Nuevos enfoques en relación al diseño y presentación de materiales de aprendizaje (Mason y Rennjie, 2008), o estrategias de aprendizaje colaborativo (Salmon, 2004; Mason y Rennjie, 2008) contribuyen a experimentar con alternativas metodológicas. Juwah (2006) ponen el énfasis en la interacción, Wenger, McDermott y Zinder (2002) en la gestión social del conocimiento.

Por nuestra parte, a partir de las investigaciones relacionadas con el proyecto EDU2008-05345 Diseño de estrategias metodológicas para el uso de Espacios Compartidos de Conocimiento mediante herramientas software y sistemas de gestión del conocimiento en entornos virtuales de formación, nos centramos en los modelos emergentes para entornos virtuales de aprendizaje (Salinas, 2009c) y aparecen junto a la configuración de los escenarios de aprendizaje (Marín y De Benito, 2011; Salinas, 
Marín y Escandell, 2011), el tema del desarrollo profesional de los docentes orientado al desarrollo de competencias docentes versatiles para poder desenvolverse en las distintas modalidades de enseñanza-aprendizaje (Gelabert, Moreno y Salinas, 2010; Salinas, 2009a; 2009b) y al mismo tiempo se prepare para facilitar un aprendizaje flexible, abierto, conectado (self organised learning).

Si analizamos la trayectoria reciente de los entornos virtuales de enseñanzaaprendizaje, podemos observar una lenta evolución, sobre todo en los cambios necesarios desde la didáctica y las metodologías a implantar, pero también una apuesta generalizada en el ámbito de la educación superior por incorporar este tipo de entornos, atendiendo tanto a las vías en las que pueden restringir o permitir ciertos tipos de interacción, como al proceso por el que las personas son capaces de construir y negociar significados a través de la interacción y la actividad colaborativa.

En este sentido, con la consiguiente actualización puede ser un buen punto de partida la propuesta que hicimos en 1995 (Salinas, 1995), al hablar de tres escenarios que venían configurados por la evolución de las redes de telecomunicaciones y de las potencialidades que aportaban a los procesos de formación: Aprendizaje en en el hogar, aprendizaje en el puesto de trabajo y aprendizaje en un centro de recursos de aprendizaje o centro de recursos multimedia.

Estos tres escenarios, que en aquel momento comenzaban a interconectarse mediante las tecnologías del momento han ido experimentando modificaciones y avances. Quizá uno de los más importantes ha sido la dotación de ubicuidad que las tecnología móviles han aportado y que dio lugar a añadirla como un cuarto escenario (Salinas, 2005b).

En efecto, los avances en la capacidad de conexión de la tecnología de uso personal teléfonos móviles, PDAs,...- hace que se vaya haciendo realidad la ubicuidad del acceso a la información y en consecuencia a los recursos de aprendizaje. Es decir el aprendizaje ubicuo, el aprendizaje en cualquier lugar/cualquier momento.

Todo ello orientado al cambio en la función de las instituciones de educación superior incorporando como una de sus misiones la educación de los estudiantes más allá del campus: desplazamiento de los procesos de formación desde entornos convencionales a otros entornos, demanda generalizada de competencias necesarias para el aprendizaje continuo, comercialización del conocimiento que genera oportunidades para nuevos mercados y competencias nuevas en el sector, etc... El ámbito de aprendizaje varía de forma vertiginosa.

\section{La agenda de investigación en TIC}

La agenda de investigación en nuestro campo, relacionada con el uso y las posibilidades de las TIC en educación, se caracteriza por experimentar un ritmo acelerado en las novedades y temas emergentes, por ofrecer un gran abanico de elementos de interés, también por estar fuertemente influenciado por las modas, y al mismo tiempo puede ocurrir que no se esté prestando la débida atención a valor y rigor de las investigaciones. 
Uno de los temas estrella de la agenda de investigación relacionada con la Tecnología Educativa lo constituyen los escenarios de aprendizaje. Desde hace unos años aparece como uno de los campos de reflexión, investigación y desarrollo más dinámicos. En los finales de los 80 comienzan a utilizarse distintas aplicaciones que paulatinamente condujeron a lo que entendemos como entornos virtuales de enseñanza-aprendizaje y que en las universidades fueron generalizándose avanzada la década siguiente. En este contexto los escenarios donde se realiza el aprendizaje se diversifican por las TIC. Y al mismo tiempo las instituciones van generando entornos basados en distintas aplicaciones de gestión (LMS). Paralelamente han surgido otros entornos alternativos o complementarios a través de las redes sociales, el surgimiento de los entornos personales de aprendizaje, etc.

Estos fenómenos han promovido abundante literatura, no toda ella suficientemente contrastada y fundamentada. También se ha desarrollado abundante investigación. Desde las areas y tematicas de investigación en tecnologia educativa constituye un ambito de investigacion que puede abordarse desde cualquiera de las areas pedagógica, organizativa y tecnológica (Salinas, 2004, 2008; Redecker, et al, 2009) o de las que Conole y Oliver (2007) señalan para la investigación en e-learning: pedagogica, organizativa, tecnología y los factores socio-culturales.

Una de las preocupaciones que deben ser preferentes es que la agenda de investigación se oriente a lograr impacto real en las políticas educativas e institucionales, en el conocimiento de cómo ocurre el aprendizaje en estos escenarios, en los cambios que ocurren en las prácticas educativas. La tendencia, sin embargo, no parece que vaya por ahí (Cabero, 2008; Zawacki-Richter, O. et al. 2009; Means et al., 2009). En este sentido Reeves (2006) caracteriza la investigación en educación preferentemente desarrollada por investigadores aislados que conducen estudios individuales, poco conectados a una potente agenda de investigación o a otras investigaciones similares, poco relacionados con la práctica y cuyos resultados no suelen llegar a los prácticos, o al menos tienen poco impacto en este terreno.

Reeves (2006)establece 5 paradigmas de investigación que componen un buen marco de referencia para los propositos de situar los escenarios de aprendizaje en la ageanda de investigación: Positivista, interpretativista, crítico, heurístico y de diseño. Se entiende aquí como paradigma al conjunto de principios teóricos y metodológicos que condicionan la visión de la realidad y la forma de orientarse para estudiarla de los investigadores. Éstos suelen asumirlos como un sistema de creencias básicas que orientan tanto la selección del fenómeno o problema investigar, la definición de los objetivos de investigación o la selección de la metodología para abordarlos Los elementos básicos de cada uno de estos paradigmas se muestran en el siguiente esquema (Fig. 1): 


\begin{tabular}{|c|c|c|c|c|}
\hline PARADIGMA & $\begin{array}{l}\text { Visión de los } \\
\text { fenómenos } \\
\text { educativos }\end{array}$ & Objetivos & Métodos & $\begin{array}{l}\text { Posición del } \\
\text { investigador }\end{array}$ \\
\hline Positivista & -Hechos objetivos & $\begin{array}{l}\text { Detectar las causas de } \\
\text { los cambios en los } \\
\text { fenómenos }\end{array}$ & Experimentales & Externa \\
\hline Interpretativo & $\begin{array}{l}\text {-Una realidad construida } \\
\text { socialmente }\end{array}$ & $\begin{array}{l}\text { Interpretar los } \\
\text { fenómenos }\end{array}$ & Etnográficos & Inmersión \\
\hline Crítico & $\begin{array}{l}\text {-Construido } \\
\text { individualmente } \\
\text { (basado en experiencia, } \\
\text { género, cultura) }\end{array}$ & $\begin{array}{l}\text { Mejorar el status de } \\
\text { los menos privilegiados }\end{array}$ & $\begin{array}{l}\text { Teoría crítica } \\
\text { Deconstrucción de } \\
\text { los fenómenos }\end{array}$ & $\begin{array}{l}\text { Implicación } \\
\text { política }\end{array}$ \\
\hline Heurístico & $\begin{array}{l}\text {-Complejo } \\
\text {-El fenómeno del } \\
\text { aprendizaje es } \\
\text { impredecible }\end{array}$ & $\begin{array}{l}\text { Proveer profesionales } \\
\text { con información para } \\
\text { la toma de decisiones }\end{array}$ & $\begin{array}{l}\text { Seleccionados en } \\
\text { base al potencial de } \\
\text { mejorar la calidad } \\
\text { de la toma de } \\
\text { decisiones } \\
\end{array}$ & Escéptico \\
\hline De diseño & $\begin{array}{l}\text {-Excesivamente } \\
\text { complejo }\end{array}$ & $\begin{array}{l}\text { Lograr impacto } \\
\text { positivo en la } \\
\text { educación y contribuir } \\
\text { al conocimiento del } \\
\text { diseño }\end{array}$ & $\begin{array}{l}\text { Diseños creativos } \\
\text { (Multiples } \\
\text { métodos) }\end{array}$ & Compromiso \\
\hline
\end{tabular}

Figura 1: Paradigmas de investigación en TIC y educación (elaborado a partir de Reeves, 2006)

Aunque con mayor o menor presencia, todos ellos son utilizados en la investigación en nuestro campo, el paradigma de la investigación basada en diseño inspira de forma preferente gran parte de la investigación relacionada con el e-learning y los escenarios de aprendizaje (van den Akker, 1999; Reigeluth y Frick, 1999; Reeves, 2000; Lee y Reigeluth, 2003; Richey y Klein, 2007). De hecho, este pardigma surge, según Anderson (2005), de la falta de impacto de la investigación educativa en el sistema educativo y se centra en el desarrollo y evaluación sistemáticos y multifacéticos de intervenciones en contextos educativos auténticos (en oposición a la investigación conducida en condiciones de laboratorio). Al igual que la investigación-acción, la investigación basada en diseño se ocupa de problemas reales identificados por los profesionales en la práctica, y por ello, el valor de este tipo de investigación debe ser medida por su habilidad para mejorar la práctica educativa, para mejorar la intervención. EL Design-Based Research Collective (2003) señala 4 áreas en las que los métodos de investigación basada en diseño presentan mayores perspectivas:

- Explorar las posibilidades para crear entornos nuevos de enseñanza-aprendizaje.

- Desarrollar teorías de instrucción y aprendizaje basadas en el contexto

- Avanzar y consolidar el conocimiento sobre diseño didáctico

- Incrementar nuestra capacidad para la innovación educativa

\section{Investigación básica vs investigación aplicada}

La investigación basada en el diseño cierra de alguna manera el debate, importante en el ámbito de la investigación relacionada con la Tecnología Educativa, sobre si debe 
realizarse investigación básica para construir teorías generalizables o dedicarse solamente a la investigación aplicada para resolver problemas específicos, concretos. No se trata tanto de una investigación empírica, cuantitativa orientada a la generalización de resultados, como de una investigación con implicaciones sobre la práctica, cuyo énfasis es la solución de problemas y la construcción de conocimiento dirigido al diseño, desarrollo y evaluación del proceso educativo, así como a desarrollar principios y orientaciones para futuras investigaciones y que cada día acumula mayor respaldo (Martínez, 1994; Cabero, 2004; Richey y Klein, 2007).

En el tema que nos ocupa entendemos que este debate es pertinente y, en este sentido, podemos considerar actual y al mismo tiempo ser clarificadora y contribuir a avanzar en nuestro campo de investigación, la matriz que Stokes (1997) propone de su visión de la investigación y que denomina Cuadrante de Pasteur (Fig. 2): la investigación se coloca en esta matriz dependiendo de si el investigador está buscando conocimiento fundamental o no y si está implicado o no con los usos prácticos de los resultados de investigación o no. Para ilustrar el esquema Stokes pone como ejemplos las investigaciones dirigidas por el físico danés Niels Bohr quién buscó conocimiento puro sobre la estructura del átomo sin implicarse en la aplicación práctica (cuadrante 1). La investigación dirigida por el inventor americano Thomas Edison, quién buscó resolver problemas prácticos mediante el desarrollo de tecnologías innovadoras mientras no expresó interés en publicar sus resultados de aprendizaje (cuadrante 3). Stokes sitúa la investigación del químico francés Louis Pasteur quién buscó conocimiento fundamental en el contexto de resolver problemas prácticos en el cuadrante 2.

A pesar de que Stokes dejó el cuadrante 4 para quienes no buscan ni conocimiento fundamental ni consideran su uso, vacío, gran parte de la investigación desarrollada en el ámbito de las tecnologías y la educación (lo mismo que otras investigaciones sobre educación) caen en el cuadrante estéril, al ser, en gran parte, desarrolladas y publicadas solamente para el avance de las carreras académicas ante la presión de publicar o perecer (Salinas y Marin, 2010).

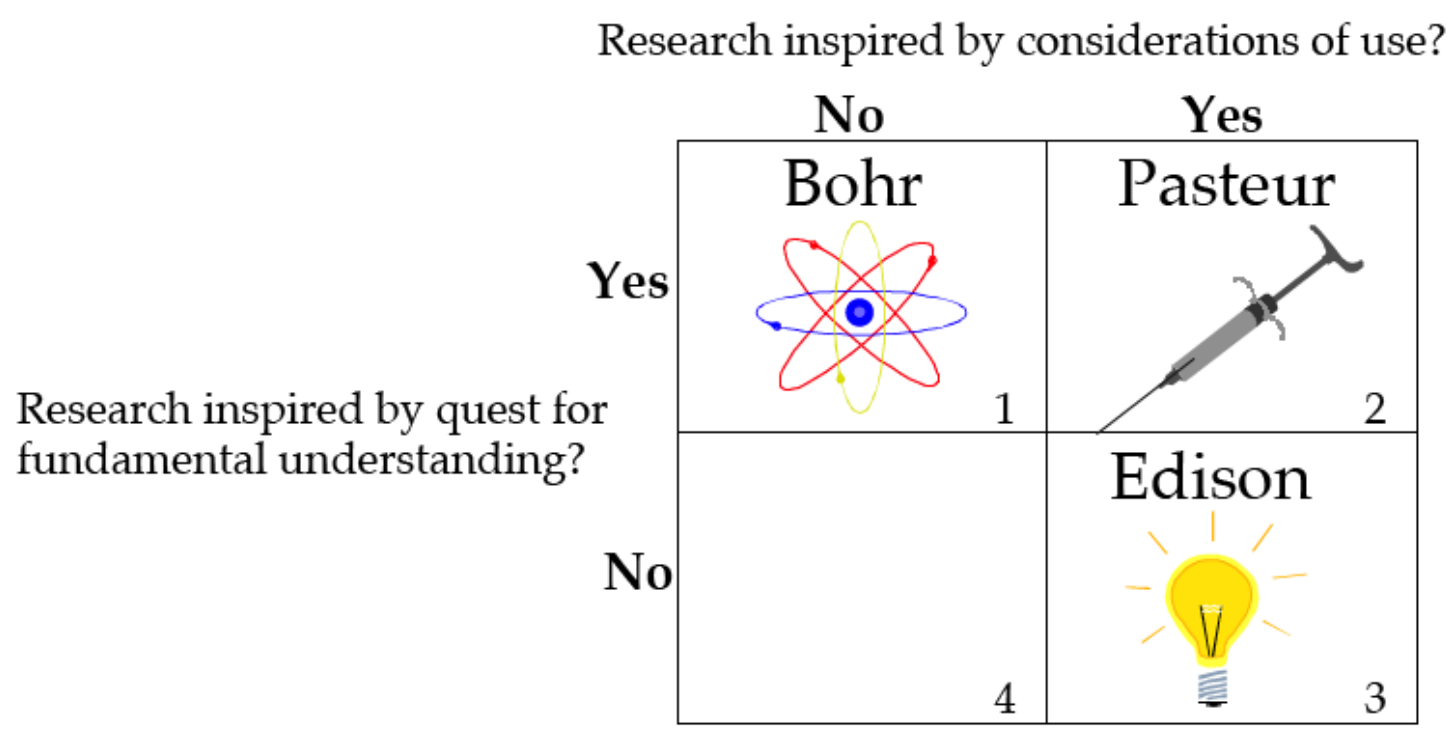

Figura 2: Cuadrante de Pasteur (Stokes, 1997) 
Atender al valor y al rigor de las investigaciones que se realizan se convierte en una de las tareas prioritarias si queremos que de verdad la investigación en el ámbito de la Tecnología Educativa tenga impacto real. O sea contribuya al avance del conocimiento pedagógico y al mismo tiempo resuelva problemas educativos.

\section{Macro-nivel y micro-nivel en las perspectivas actuales de investigación en TIC y educación}

Nuestro ámbito de estudio ofrece gran cantidad de temas de investigación, los fenómenos y problemáticas relacionados con el aprendizaje y el uso de las TIC ofrecen multiples perspectivas de observación, experimentación e intervención.

Uno de los temas preferentes de investigación, ya señalado, lo constituyen los nuevos escenarios de aprendizaje que se configuran por la evolución de las tecnologías de red. Como objeto de estudio, los escenarios de aprendizaje responden a una de las características de la investigación sobre TIC y educación: la interdisciplinariedad. Tanto se investiga sobre el uso de paquetes de software de uso educativo, como características de los entornos virtuales multi-usuario o la influencia de las redes sociales. Desde la perspectiva pedagógica, los fenómenos que se dan en las situaciones educativas presentan cuatro manifestaciones que podemos considerar como respuestas desde la práctica, de gran interés para comprender el fenómeno y todas ellas interrelacionadas dentro de los procesos de innovación:

- Cambios en el rol del profesor,

- Cambios en el rol del alumno,

- Cambios en el proceso de enseñanza-aprendizaje (cambios metodológicos)

- Implicaciones políticas, sociales, institucionales.

Los cambios en las prácticas, en la forma de desenvolverse de formadores y alumnos en estos nuevos espacios comunicativos pueden ser considerados como verdaderos cambios de su rol en el proceso de enseñanza-aprendizaje.

Si el análisis lo enfocamos desde los aspectos metodológicos de las experiencias didácticas convendremos en que no pueden analizarse de forma aislada y así van a sufrir condicionamientos tecnológicos (enseñanza mediada, avances y configuraciones de nuevos entornos, etc.), condicionamientos que provienen del contexto organizativo donde se desarrollan estos procesos, o de los medios que se están empleando, la forma en que representan el conocimiento, etc.. y todos ellos vienen condicionados por los modelos pedagógicos (nuevos o clásicos adaptados en los que nos basamos..) (Salinas, 2005a).Cada uno de estos aspectos puede abordarse desde distintas perspectivas o desde distintos niveles. Conole y Oliver (2007) estabecen una perspectiva macro y otra micro, Zawacki-Richte y Bäcker (2009) incluyen una tercera meso. Esta propuesta coincide con la que se estabece en Salinas (2005a) para la gestión de los Entornos Virtuales de Formación, dando lugar a tres niveles o tipos de gestión de los mismos: Estrategias de introducción y/o implementación; estrategias de implantación y diseminación en la institución; y de práctica y experiencia diaria). Cada uno de ellos implica diferentes tipos de responsabilidades en el momento de tomar decisiones y llevar a cabo procesos de mejora concernientes a uno u otro nivel. 
A la hora de plantear y desarrollar una investigacion, conviene tener claro en todo momento en el nivel en el que nos situamos. No resulta igual, ni requiere los mismos recursos una investigación a nivel institucional o suprainstitucional, que si se pretende resolver problemas en un contex to determinado.

Puede ser clarificador atender a los temas de investigación que Conole y Oliver (2007) situan en cada una de las perspectivas de investigación en relación al e-learning:

- Macronivel: hacen referencia al nivel institucional

- Conocimiento, sociedad y perspectivas de las TIC en el aprendizaje

- Crítica del impacto de las políticas y financiación

- Teorías del aprendizaje y su aplicación al e-learning

- El diseño de tecnologías para el aprendizaje

- Impacto del e-learning en estructuras y roles organizacionales

- Micronivel: hacen referencia a práctica y experiencia

- Diseño de experiencias de aprendizaje

- Diseño de recursos digitales para el aprendizaje

- Gestión de los recursos educativos

- Aspectos afectivos y sociales de las TIC

- Alfabetización académica para el s:XXI

- Colaboración

- Evaluación

¿Cuáles son las situaciones problemáticas a las que nos estamos enfrentando? Sin duda que cualquiera de los temas señalados anteriormente requiere de estudio e investigación, pero son sin duda los escenarios donde se produce el aprendizaje, los distintos entornos que pueden configurarse uno de los temas más atractivos y relevantes, y que de una forma $\mathrm{u}$ otra pueden considerarse como un tema transversal a todos ellos.

\section{Los escenarios de aprendizaje futuros como objeto de investigación}

Para comprender mejor la configuración futura de los entornos de aprendizaje puedes ser útil lo que Rhodes (1994) denomina la construcción de escenarios. Un escenario viene a ser la descripción, en detalle, de lo que estamos concibiendo o imaginando y de lo que significaría, llevado a la realidad, para un grupo o situación particular. En educación los escenarios suelen describir un día o una situación concreta de estudiante o de profesor en un contexto educativo del futuro, y el proceso de creación de estos escenarios ayuda a los implicados en la planificación del cambio a que tengan una mejor comprensión de todo el proceso.

Describir escenarios de aprendizaje propiciados por las tecnologías de red nos ayudará en el diseño y creación de ambientes de aprendizaje adecuados a las nuevas coordenadas espacio-temporales, a las nuevas modalidades educativas, a los nuevos perfiles de los actores, de tal forma que podamos comprender cómo los cambios afectan a los estudiantes, profesores, centros y a la comunidad.

Estos nuevos escenarios pueden referirse, tanto al impacto que la evolución de las TIC tiene y seguirá teniendo en los entornos virtuales 'convencionales', como a la 
configuración de nuevos escenarios para el aprendizaje, considerados en una proyección de futuro.

La construcción de escenarios sigue variados procedimientos de trabajo. Un buen esquema de trabajo que encaja bien en la investigación basada en diseño, lo constituye la propuesta de Henning (2009) para acercarse a la construcción de escenarios en 3 fases: $1^{\text {a }}$ Planificación creativa del escenario para un futuro lejano; $2^{\mathrm{a}}$ Un proceso de pensamiento inverso orientado a la resolución de problemas que podrían plantearse en un futuro cercano; y $3^{\mathrm{a}}$ Un proceso de resolución de problemas de este futuro cercano a partir de la situación actual (Fig. 3).

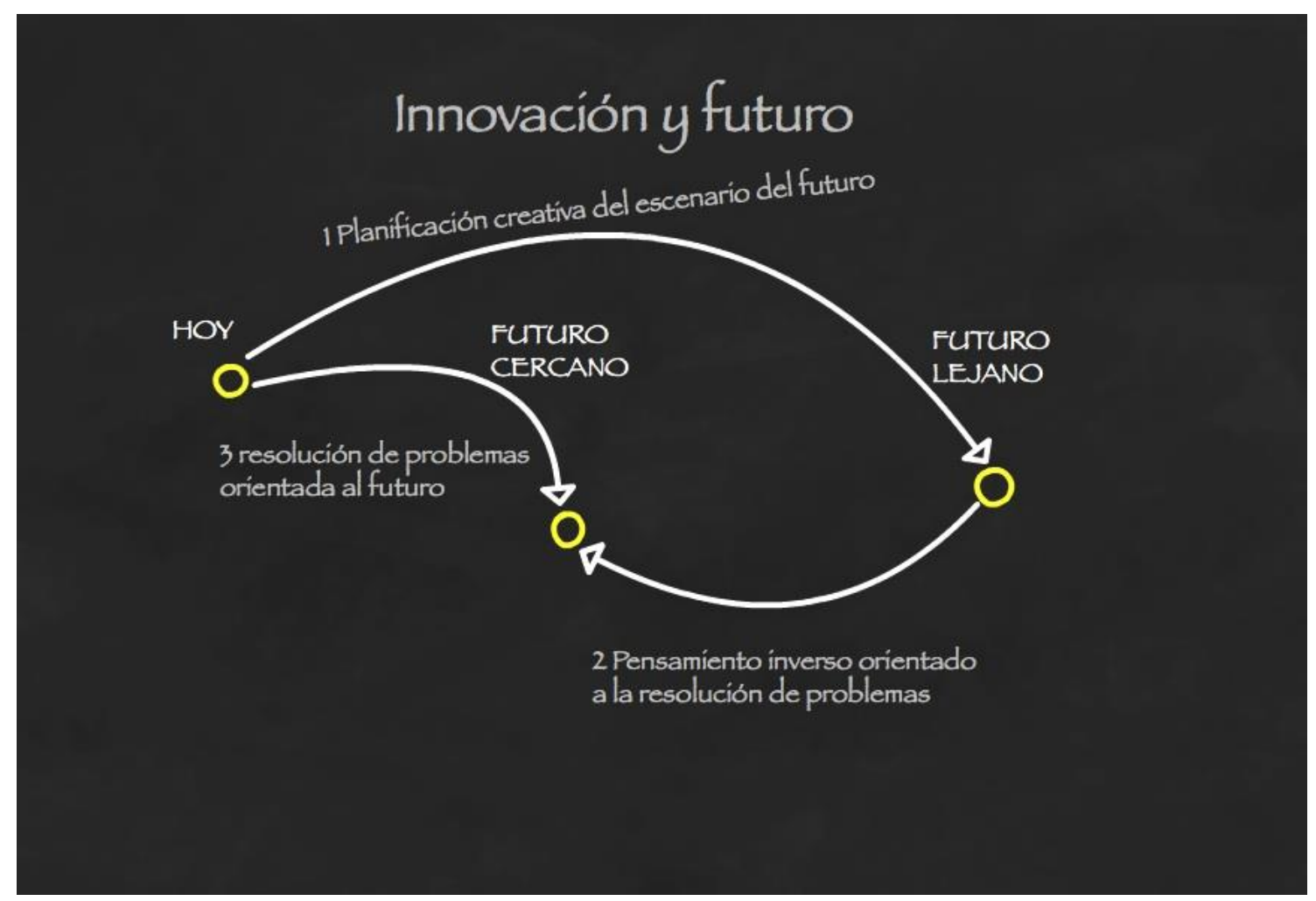

Figura 3.- Esquema del procedimiento de construcción de escenarios (basado en Henning, 2009).

En todo caso, es difícil proyectar (o imaginar) el futuro del aprendizaje sin partir del conocimiento profundo del presente y de las prácticas actuales. No es conveniente ignorar las nuevas posibilidades y aportaciones tecnológicas, como tampoco lo es olvidarse de la tradición y el saber pedagógico desarrollado durante el pasado siglo. Por eso, caer en la tentación de las dicotomias que enfrentan la educación prehistórica (a.d. Web 2.0) y la fetén (d.d. Web 2.0), tan frecuentes en nuestro ámbito, no aporta gran cosa al proceso de análisis crítico y de propuestas innovadoras. Hay que partir de la idea de que el objeto de estudio, en lugar de blanco o negro, presenta casi siempre una gama de grises, y queuna de las leyes invariables de la tecnología es que todo nuevo sistema debe coexistir con los sistemas anteriores.

La construcción de escenarios futuros representa un conjunto de investigaciones en la intersección de tres perspectivas: 
- de la formación continua (Life Long learning) al ocuparse de procesos destinados a la formación académica, profesional, personal, cívica y social de la persona, así como aquellos orientados al empleo;

- del e-learning y los entornos virtuales de aprendizaje apoyados en redes, tanto los entornos institucionales, el software social, los entornos personales de aprendizaje, junto a los elementos de adecuación de los entornos al alumno

- de los procesos de generación e intercambio de conocimientos (aprendizaje social, desarrollo profesional continuo,...).

En este terreno, no abundan referentes para el campo educativo; Brown (2005); Rudd et al. (2006); Punie et al. (2008); Anderson et al. (2012) pueden ser algunos de ellos. Como ejemplos, pueden señalarse los trabajos sobre 'Learning Networks' del Educational Technology Expertise Centre de la Open University of the Netherlands (Koper y Tattersall, 2004; Koper, Rusman y Sloep, 2005). Otro ejemplo de este tipo lo constituyen el SKG Project (Souter et al, 2011) yLearning in Networks of Knowledge (LINK) (Allen, 2011) auspiciados por el Australian LEarning \& Teaching Council.

Un caso paradigmático podría ser Futurelab que desarrolla diversos trabajos de prospectiva. Puede servir como ejemplo el proyecto VisionMapper (Futurelab, 2009), que presenta una propuesta de 6 escenarios futuros. Esta propuesta, fruto del trabajo de un equipo de 98 expertos para proporcionar un poderoso recurso para la planificación educativa, incluye:

- 1.- El escenario de la elección informada. La educación como trayectoria personal diseñada en colaboración con los mentores para aprender a satisfacer las necesidades personales y tener en cuenta su historial a lo largo de la vida.

- 2.- El escenario de consumidores independientes. La educación como mercado diverso donde las personas pueden elegir entre una amplia gama de diferentes ofertas educativas estandarizadas.

- 3.- Descubrimiento. La educación consiste en identificar la contribución específica que se puede realizar en diferentes organizaciones y comunidades, y saber donde va a surgir.

- 4.- El escenario de diagnóstico. La educación diagnostica el potencial del estudiante, dicta su lugar en la organización y traza su futuro. Aquí es donde usted encaja. Manos a la obra.

- 5- El escenario de experiencia integrada. La educación se caracteriza por la participación en una serie de prácticas auténticas a través de las diversas comunidades

- 6.- El escenario de servicios y ciudadanía. La educación es una institución social independiente que se centra en el desarrollo de las competencias genéricas y las competencias para participar en las comunidades y en una esfera social más amplia.

La búsqueda de entornos más adecuados para la gestión del aprendizaje desde una perspectiva institucional también puede ser considerada como un caso particular de construcción de escenarios. En este terreno, pueden destacarse los trabajos deAttwell (2007), Wilson et al. (2009); Santos y Pedro (2010); Casquero et al. (2008, 2010); White y Davis (2011), Buchem, Attwell y Torres (2011), entre otros. 


\section{En busca de los nuevos escenarios de aprendizaje: configuraciones y competencias docentes}

En el diseño de cada uno de los escenarios lo fundamental no es la disponibilidad tecnológica, también debe atenderse a las características de los otros elementos del proceso didáctico y en especial al usuario del aprendizaje. La investigación debe atender, entocnes, tanto al estudio de las configuraciones tecnológicas, como la las competencias para desenvolverse en las mismas de los actores.

\section{De los EVEA a los iPLE}

Los entornos virtuales de enseñanza-aprendizaje que utilizan las instituciones y que podemos caracterízar como escenarios de la web 1.0 se construyen sobre aplicaciones de gestión para la gestión de los entornos virtuales de aprendizaje (los llamados LMS).

Desde la perspectiva pedagógica a pesar de que supusieron avances y dieron lugar a muchas experiencias innovadoras, han estado siempre preferentemente orientados a la producción y, sobre todo, a la distribución de contenido.

Dejando de lado el abanico de posibilidades que incorporan a los procesos formativos formales, hay que señalar que estas aplicaciones para la gestión de los entornos virtuales de aprendizaje, sean comerciales o de software libre, se centran primeramente en la administración del curso antes que en la interacción profesor-alumno / alumno-alumno. Pocas de las aplicaciones integradas -LMS- han sido diseñadas desde una visión sistémica fundada en las teorías del aprendizaje. Las más usuales responden bien cuando el aprendizaje se define en términos de transmisión de conocimiento, ya que el elemento central es el diseño del contenido más que el diseño del proceso de aprendizaje. Esto se corresponde muy bien con la práctica habitual en los EVEA de poner contenido en web y que permite utilizar estrategias didácticas muy planificadas y predefinidas. Pero si se define el aprendizaje como construcción de conocimiento en lugar de transmisión, entonces el aprendizaje se convierte en una actividad que principalmente proporciona significados y en la que el estudiante busca construir una representación mental coherente a partir del material presentado. En estos casos, se observan limitaciones por parte de las distintas plataformas, sean de software propietario o sean de software libre (De Benito y Salinas, 2008;Salinas, 2009a).

En efecto, los EVEA institucionales replican (la percepción de) la enseñanza tradicional mediante la distribución de contenidos on-line, permitiendo la creación y distribución de contenido, el envío de correos y anuncios y la comunicación mediante chats y foros. Todo ello en un entorno seguro y controlado (desde le punto de vista del profesor).

Estas limitaciones presentes en los entornos virtuales institucionales, en algún caso parecen debidas a un cierto estancamiento en su utilización -masa crítica en número de cursos, penetración real en las actividades formativas, compromiso de formadores y estudiantes, modelos didácticos inadecuados, etc.-, pero en gran parte están asociadas a la rigidez de las plataformas para adaptarse a nuevos modelos que orienten el proceso de formación (Salinas, 2009a). Como limitaciones pueden destacarse:

- Incorpora modelos obsoletos de enseñanza 
- Situa al profesor en el centro del procesoen lugar de al alumno

- No se integra con las herramientas y entornos que usan habitualmente alumnos y profesores

- Son fundamentalmente cerrados (no atienden al aprendizaje en red)

- Aislan a los actores del exterior

- No motivan a los alumnos a tomar la responsabilidad sobre su propio aprendizaje, sobre las herramientas y sobre la alfabetización digital.

Estos entornos virtuales de enseñanza-aprendizaje, que etiquetamos como 'institucionales', incorporan este tipo de componentes tecnológicos, pero sobre todo componentes organizativos y pedagógicos. También incorporan limitaciones para el despliegue de metodologías centradas en el alumno.

Los avances tecnológicos y los cambios en los usos sociales de las tecnologías de red, han propiciado la diversificación de espacios de comunicación y aprendizaje. Así, suele hablarse de entorno social de aprendizaje, que apoyado en una rápida evolución del software social está permitiendo nuevas formas de interacción y comunicación más horizontal, y que configuran otros entornos como redes sociales y comunidades virtuales que toman cada vez mayor importancia. Aunque en muchos casos responde más a un fenómeno de moda en la red y debe ser considerado con precaución, es indudablemente que aportan grandes posibilidades de formación y por ello debe ser abordado desde la investigación. Aquí, parece necesario estudiar el fenómeno en cuanto que canaliza variadas formas de aprendizaje no formal y sobre todo informal.

En la intersección de ambos fenómenos pueden situarse los entornos personales de aprendizaje, que se presentan como un sistema bisagra donde integrar el entorno virtual institucional en el que estamos distribuyendo cursos y asociado preferentemente al aprendizaje formal, y este entorno más informal que ofrecen redes sociales y comunidades virtuales de aprendizaje para construir las propias Redes Personales de Conocimiento (Personal knowledge Network PKN)(Chatti, 2009; Salinas, 2009c).

Si el entorno social y el personal se dan sobre todo en el ámbito informal, los entornos institucionales se situan todavía en el ámbito formal. Encontramos tendencias a integrar lo formal, no formal e informal. Entre ellas encontramos trabajos que se ocupan del estudio y del desarrollo de artefactos y experiencias que integren los distintos entornosmediante iPLE: Wilson et al. (2009); Taraghi, Ebner, y Schaffert, (2009); Santos y Pedro (2010); Casquero et al. (2010); White y Davis (2011). En nuestro caso, los primeros avances se han desarrollado de acuerdo a lo que hemos dado en llamar Modelo de Aprendizaje Flexible(provisional) (fig. 4) (Salinas, Marin y Escandell, 2011). 


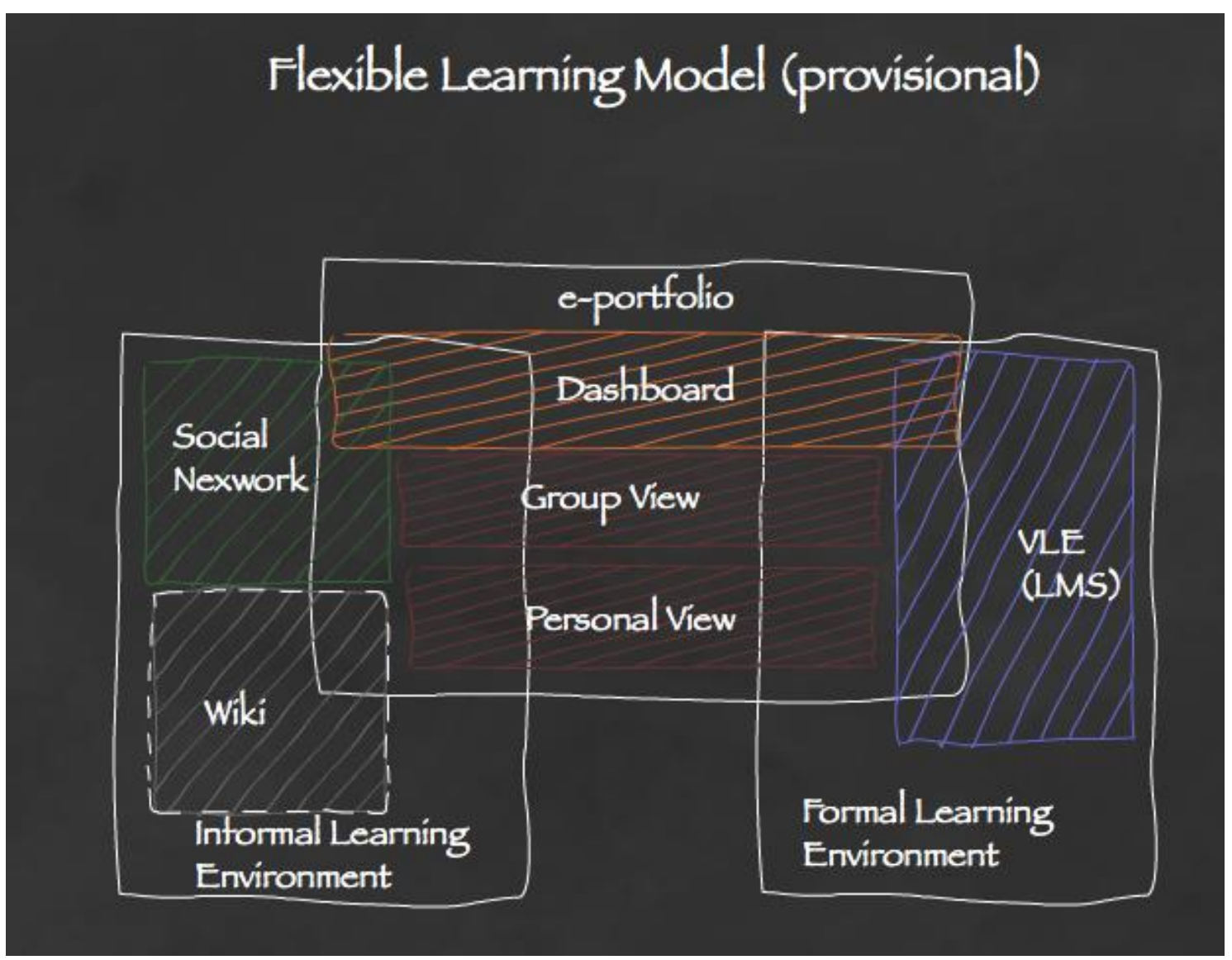

Figura 4.- Modelo de Aprendizjae Flexible (provisional) (Salinas, Marin y Escandell, 2011)

Estos planteamientos pretenden, en esencia, incorporar aspectos del ambito informal y no formal en los entornos formales, al mismo tiempo que facilitan el uso de metodologías centradas en el alumno, y para ello lo hacen desde una concepción de las instituciones educativas como instituciones de gestión del conocimiento. Integración facilitada por el uso de redes sociales que pueden superar los límites institucionales, y, sobre todo, por el uso de los nuevos protocolos de red (P2P, servicios web, cloud, sindicación, etc.) para conectar un rango de recursos y sistemas en un espacio gestionado personalmente. La idea responde bien al concepto de ecología de aprendizaje (Siemens, 2006), entendiendo como tal un entorno que apoya y promueve el aprendizaje y que puede caracterizarse por ser adaptativo, dinámico, auto-organizado / dirigido individualmente; estructurado informalmente; diverso; vivo.

Para nosotros estos nuevos entornos se caracterizan por incorporar posibilidades de personalización del entorno desde la visión del estudiante y también del profesor, por la modularización del entorno de modo que cada profesor pueda incorporar aquello que se adecua mejor a sus planteamientos metodológicos y por requerir nuevas competencias de los actores como ya se ha señalado.

Una de ellas que se hace imprescindible viene a ser la gestión personalizada de la información. En efecto, el aumento de la autonomía del alumno añade, a la superación de las barreras de la distancia y el tiempo para acceder al aprendizaje, mayor interacción y la oportunidad de compartir el control de las actividades de aprendizaje mediante la intercomunicación en un marco de apoyo y colaboración. Y entre estos aspectos, 
consideramos que debe atenderse a los desarrollos relacionados con la gestión personal de la información (Personal Information Management, PIM) como uno de los procesos clave en los sistemas de gestión del conocimiento en el ámbito del aprendizaje.

La integración de los entornos virtuales de aprendizaje en este marco contribuye a invertir la tendencia de alumnos adaptándose al sistema para ir hacia un sistema cada vez más adaptado al alumno. En la línea de Mott y Wiley (2009) al proponer la red de aprendizaje abierto (OLN), un híbrido de CMS y el ambiente de aprendizaje personal (PLE), la idea es avanzar en una alternativa a los entornos institucionales para aprovechar las potencialidades de la Web en la mejora del aprendizaje.

Todo esto responde a conceptos con cierta tradición que hacen referencia al aprendizaje abierto (Lewis y Spencer, 1986) y a los modelos de educación flexible (Van Den Brade, 1993; Tait, 1999; Moran y Myringer, 1999; Salinas, 1999; Collis y Moneen, 2001; Fallows y Bhanot, 2002). Independientemente de si la enseñanza es presencial o a distancia atribuyen al alumno la posibilidad de participar activamente en la toma de decisiones sobre el aprendizaje y supone una nueva concepción tanto en la organización administrativa, como de los materiales y sistemas de comunicación y mediación, y sobre todo, de las metodologías a implantar.

\section{Las competencias docentes para los nuevos escenarios}

No puede separarse el análisis de los escenarios de aprendizaje de las competencias necesarias para desenvolverse en ellos en la práctica del proceso e-a de los actores, especialmente en los profesores (Marin et al., 2007, 2012; Couros, 2010; Castañeda y Adell, 2011).

Los escenarios de aprendizaje situados entre la web 1.0, el blended learning y los escenarios del futuro, requieren ya pericia, por parte de los profesores, en relación a:

- los modelos de puesta a distancia de la formación

- los dominios de la producción y distribución de contenidos y de recursos a distancia

- los efectos psico-sociológicos de los dispositivos síncronos y asíncronos sobre el aprendizaje

- $\quad$ sobre los mismos dispositivos

Atendiendo a estos aspectos puede entenderse que los avances en los modelos de actualización del profesorado se convierten en un tema de investigación inseparable del diseño y desarrollo de nuevos escenarios. Disponemos de modelos surgidos de la investigación (TPACK sería un caso emplemático), pero puede ser necesario seguir trabajando en este terreno para no perder el paso de los avances tecnológicos.

Alumnos y profesores necesitan adquirir habilidades con el sistema, pero sobre todo con la modalidad comunicativa. Así, requieren, entre otras cosas, guía para avanzar en los límites de nuevos espacios comunicativos, de nuevos entornos de formación; discriminar entre los espacios de comunicación; control sobre la dinámica comunicativa; normas de regulación del grupo, etc.. 
Estos desafíos están requiriendo un perfil permanentemente en cambio de los docentes. Se trata de manejarse en ambientes que al mismo tiempo que incorporan estos tipos de aprendizaje van a requerir nuevas competencias para manejarse en el e-learning:

- Un e-learning inclusivo: Maestría de los dispositivos de aprendizaje en entornos digitales, en el proceso de trabajo

- Un e-learning extensivo: maestría de la ergonomía cognitiva sobre soportes móviles, en entornos físicos de transición

- Un e-learning contributivo: Maestría de las dinámicas de contribución y de reputación digital en las redes sociales.

Se trata de un docente caracterizado por la conectividad que facilita el manejo adecuado de la 'affordance' pedagógica, un nuevo manejo del conocimiento y la construcción de redes /entornos personales de aprendizaje. Para Wheleer (2011) este docente estaría caracterizado por:

- La 'curación' del contenido (filtro, manejo,...)

- La colaboración

- El co-aprendizaje

- La facilitación

- El apoyo al aprendizaje

- La inspiración

Cualquier avance hacia nuevos escenarios va a requerir cierta expertise de los profesores respecto a las competencias pedagógicas asociadas. $\mathrm{Si}$ pensamos la enseñanza como diseño de situaciones y experiencia de aprendizaje, como guía y facilitación del uso de recursos y herramientas que necesitan para explorar y elaborar nuevo conocimiento y destrezas, actuando el profesor como gestor de la pleyade de recursos de aprendizaje y acentuando su papel de orientador en lugar de entenderla como mera transmisión mecánica de contenidos, (Salinas, Perez y De Benito, 2008) entonces se va a requerir manejarse con soltura en los distintos modelos de puesta a distancia, cierta maestría en la producción y distribución de contenidos y recursos para situaciones diversas (blended, distancia, etc..); dominio de distintos aspectos relacionados con los dispositivos, así como conocimiento de los efectos de estos dispositivos sobre el aprendizaje. Paradójicamente, en estas metodologías centradas en el alumno el papel del formador presenta una mayor complejidad.

\section{Los desafíos}

Tanto desde el terreno de los escenarios de aprendizaje, como del de los modelos y desarrollo de competencias pedagógicas para el mundo digital, la actual situación de investigación se enfrenta a distintos desafíos.

Desafíos que provienen de una nueva forma de entender el aprendizaje a lo largo de la vida, a lo largo del trabajo, y con los otros (en un mundo digital). Es decir, el futuro próximo se está caracterizando por un aprendizaje embebido, continuo y basado en el aprendizaje social: 
- Aprendizaje embebido, en cuanto cada vez toma mayor importancia la formación a lo largo del trabajo, dentro del trabajo si solución de continuidad.

- Aprendizaje continuo, asociado a una gestión personal del aprendizaje, sin solución de continuidad en el tiempo y en el espacio, disminuyendo la diferencia entre vivir, trabajar y aprender.

- Aprendizaje social, desde el momento que existe una organización colectiva y contributiva del aprendizaje, con valorización de los aportes y del apoyo entre pares.

Se trata, en definitiva, de un aprendizaje caracterizado por una creciente porosidad, aspecto importante desde el punto de vista de la investigación que se presenta entre distintos escenarios, y que puede observarse entre lo real y lo virtual, entre jugar y aprender, entre formarse e informarse (o mejor gestionar información), entre vivir, trabajar y aprender.

Los iPLE requieren imaginar nuevos espacios comunicativos que puedan albergar los distintos tipos de aprendizaje. Al mismo tiempo que incorporan estos tipos de aprendizaje van a requerir nuevas competencias para manejarse en los mismos. Abordados desde la investigación vamos a encontrar temas que no son nuevos, pero que adquieren nueva importancia en este contexto de la comunicación e interacción a caballo entre contextos presenciales y digitales, al contribuir a que el estudiante acomode sus formas de comunicación, se apropie y domine el nuevo entorno, para que se produzca el aprendizaje, la construcción personal del conocimiento, la realidad del conocimiento compartido.

Los desafíos que estamos describiendo ponen en el centro de la agenda de investigación el estudio, la reflexión, el diseño y el desarrollo de entornos de aprendizaje, el cambio de modelos en la actualización de los profesores que den cabida a este nuevo perfil docente. Pero, al mismo tiempo, se observa la necesidad de propuestas y conocimiento de las metodologías centradas en el alumno en estos entornos virtuales y la búsqueda de un nuevo modelo pedagógico que se ajuste a la concepción de las instituciones como instituciones de gestión de conocimiento.

Es imprescindible avanzar desde los modelos que describen la enseñanza como un "proceso técnico" y que contempla al profesor como un simple ejecutor al que hay que equipar de competencias y habilidades para aumentar su eficacia por medio de los recursos (desplegando lo que se ha denominado metodologías 'genéricas'), hacia modelos más abiertos que ven la enseñanza como un espacio de saber y conocimiento y espacio sociopolítico en el que el conocimiento se selecciona, legitima y distribuye a los sujetos diferencialmente y que ve al profesor como un profesional dotado de capacidad de decisión y juicio y capaz de reconstruir su propia práctica críticamente y de incluir los medios de un modo creativo (metodologías 'específicas') (Salinas, 2009c).En definitiva, con las estrategias centradas en el alumno se trata de motivarlos a aprender de una forma nueva y poco familiar, y en el caso del aprendizaje en red, utilizando un abanico de herramientas y técnicas muy diversas y, a veces, poco conocidas (Salinas, 2004; Prendes, 2007).

Para poder incorporar metodologías centradas en el alumno, metodologías más artesanales desde la perspectiva del docente, dichos entornos tendrán que responder a planteamientos abiertos, flexibles como se ha señalado. Se espera que en los EVEA 
abiertos puedan generarse propuestas curriculares y didácticas flexibles adaptables a las características del usuario, ampliando su conocimiento y estimulando la investigación y la autonomía del alumno. Es decir, que potencien la interacción, la conversación y el aprendizaje social, el desarrollo profesional y personal continuo y establezca conexiones a nivel global.

Es imprescindible, por tanto, atender al modelo de enseñanza-aprendizaje que subyace, y esto requiere una nueva mirada sobre los modelos pedagógicos, un fuerte apoyo de tecnologías, cambios importantes en la organización tanto administrativa, como de los materiales y sistemas de comunicación y mediación.

No tiene sentido hablar de metodologías centradas en el alumno sin considerar el protagonismo de éste en su propio proceso de aprendizaje -núcleo central de la educación flexible ya señalado en el capítulo primero al hablar del control-y en el conjunto de decisiones en las que se ve implicado (Salinas, 2004). Las estrategias didácticas centradas en el alumno se inclinan más hacia éste último y representan alternativas a partir de las cuales el profesor puede elegir una nueva metodología de enseñanza basada en el trabajo activo, en la autonomía y en la flexibilidad, donde el alumno sea el protagonista de su formación.

En este contexto consideramos relevante investigar sobre las posibilidades que la integración de los sistemas para la gestión del conocimiento ofrecen para desarrollar nuevas modalidades en los procesos de e-a en entornos virtuales. Y la pregunta a plantearse sería ¿cómo logramos la adecuada combinación de elementos pedagógicos, tecnológicos y organizativos del escenario de aprendizaje que estamos construyendo? La preocupación es, entonces, de corte metodológico: la idea es que se logra mayor calidad desplegando aquellas estrategias didácticas que mejor respondan a las características del usuario, al conocimiento con el que estamos trabajando, a la organización y al contexto donde nos movemos, utilizando herramientas software que faciliten la interacción y estructuras de información y conocimiento.

Todo esto requiere procesos de experimentación y validación de herramientas software, pero también de metodologías y formas de implantación de sistemas de enseñanza flexible. Por ello, para lograrlo necesitamos conocer, experimentar y validar -en su componente pedagógica- las distintas herramientas y recursos susceptibles de configurar los nuevos escenarios de aprendizaje.

Concluida la versión original el 15 de Septiembre de 2012.

Aprobado el 20 de Septiembre de 2012.

Publicado el 15 de Julio de 2016.

Salinas, J. (2016). La investigación ante los desafíos de los escenarios de aprendizaje futuros. RED. Revista de Educación a Distancia, 50. Consultado el (dd/mm/aaaa) en http://www.um.es/ead/red/50 


\section{Referencias}

Allen, M. (2011). Learning in Networks of Knowledge: improving student outcomes using Web 2.0 concepts and a knowledge-networking approach. Australian Learning and Teaching Council, Strawberry Hills. Recuperado de: http://knowledgenetworklearning.net

Anderson, I.Q., Boyles, J.L., Rainie, L. (2012). The future impact of the Internet on higher education: Experts expect more-efficient collaborative environments and new grading schemes; they worry about massive online courses, the shift away from oncampus life. Pew Research Center's Internet \& American Life Project, Washington, D.C. Recuperado de: http://www.pewinternet.org/topics/Future-of-the-internet.aspx

Anderson, T. (2005). Design-based research and its application to a call center innovation in distance education. Canadian Journal of Learning and Technology, 31(2), 69-84. Recuperado de: http://www.jofde.ca/index.php/jde/article/view/18/552

Attwell, G. (2007). The Personal Learning Environments - the future of eLearning? In: eLearning Papers. 2(1). Recuperado de: http://www.elearningeuropa.info/files/media/media11561.pdf

Brown, M. (2005). Learning spaces. En D. Oblinger \& J. L. Oblinger (Eds.), Educating the net generation. Recuperado de: http://www.educause.edu/educatingthenetgen/5989

Buchem, I., Attwell, G., Torres, R. (2011). Understanding Personal Learning Environments: Literature review and synthesis through the Activity Theory lens. In Proceedings of the The PLE Conference 2011 (pp. 1-33), 10th - 12th July 2011, Southampton, UK. URL. Recuperado de: http://journal.webscience.org/658/

Cabero, J. (2004). La investigación en Tecnologías de la Educación. Bordón, 56(3-4), 617-634.

Cabero, J. (coord) (2008). E-learning: metaanálisis de investigaciones y resultados alcanzados. EA2008-0134, Dirección General de Universidades, Madrid.

Casquero, O., Portillo, J., Ovelar, R., Benito, M. and Romo, J. (2010). iPLE Network: an integrated eLearning 2.0 architecture from a university's perspective. Interactive Learning Environments, 18(3), 293-308. Recuperado de: http://dx.doi.org/10.1080/10494820.2010.500553 (Última consulta: 27/4/2011)

Casquero, O., Portillo, R., Ovelar, R., Romo, J. and Benito, M. (2008). iGoogle and gadgets as a platform for integrating institutional and external services. Universidad del País Vasco. In Workshop on Mash-Up Personal Learning Environments (MUPPLE'08). Recuperado de: http://citeseerx.ist.psu.edu/viewdoc/download?doi=10.1.1.143.708\&rep=rep1\&type= pdf 
Castañeda, L. \& Adell, J. (2011). El desarrollo profesional de los docentes en entornos personales de aprendizaje (PLE). En Roig, R. y Laneve, C. La práctica educativa en la sociedad de la información. Innovación a través de la investigación. La pratica educativa nella società dell'informazione. L'innovazione attraverso la ricerca. Alcoy (España)-Brescia (Italia): Editorial Marfil \& La Scuola Editrice. Recuperado de:

http://digitum.um.es/xmlui/bitstream/10201/24647/1/CastanedaAdell2011preprint.pd $\mathrm{f}$

Chatti, M. A. (2009). Mashup Personal Learning Environment. http://www.google.com/reader/shared/06179808011277023861 10/4/2010

Collis, B. y Moneen,J. (2001). Flexible Learning in a digital world. Kogan Page, London.

Conole, G. (2008). Capturing practice: The role of mediating artefacts in learning design. In L. Lockyer, S. Bennett, S. Agostinho \& B. Harper (Eds.), Handbook of research on learning design and learning objects: Issues, applications and Technologies (pp. 187-207). Hersey: IGI Global.

Conole, G., Oliver, M. (Ed.)(2006). Contemporary perspectives in E-learning Research. Themes, methods and impact on practice. New York: Routledge.

Couros, A. (2010). Developing Personal Learning Networks for Open \& Social Learning. In Veletsianos, G. (Ed). Emerging Technologies in Distance Education. Edmonton: Athabasca University Press. Retrieved fromhttp://www.aupress.ca/books/120177/ebook/06_Veletsianos_2010Emerging_Technologies_in_Distance_Education.pdf

De Benito, B. (2006). Diseño y validación de un instrumento de selección de herramientas para entornos virtuales basado en la toma de decisiones multicriterio. Tesis doctora inédita. Universitat de les Illes Balears, Palma de Mallorca

De Benito, B., y Salinas, J.(2008). Los entornos tecnológicos en la universidad. Pixel Bit. Revista de Medios y Educación. 83 - 101.

Fallows, S. y Bhanot, R. (2002). Educational Development Through Information and Communications Technologies. Kogan Page, London

FutureLab (2009). 6 Future Scenarios. VisionMapper. http://www.visionmapper.org.uk (Recuperado 25/10/2011)

Gelabert, J.; Moreno, J., y Salinas, J. (2010). Construcción de Entornos Personales de Aprendizaje por profesores universitarios. The PLE Conference. Barcelona.

Henning, T. (2009). Innovation and the future. Brand Worshop Basisdesign, Berlin

Juwah,C. (2006). Interactions in Online Education (Open \& Flexible Learning). Open $\&$ Flexible Learning series. London: Routledge. 
Koper, R., (2004). Learning technologies in e-learning: An integrated domain model. In Jochems, W., Van Merrienboer, J., \& Koper, R. (Eds.), Integrated e-Learning: Implications for Pedagogy, Technology and Organization (pp. 64-79). London: Routledge.

Koper, E.J.R., Rusman, E., Sloep, P. (2005). Effective Learning Networks. Lifelong learning in Europe, 1, 18-27.

Koper, R.; Tattersall, C. (2004). New directions for lifelong learning using network technologies. British Journal of Educational Technology, 35(6), 689-700.

Marín, V. \& De Benito, B. (2011). A design of a postgraduate course on Google Apps based on an Institutional Personal Learning Environment (iPLE). The PLE Conference 2011. Southampton, UK. Retrieved from http://journal.webscience.org/652/

Marín, V., y Romero, A. (2007). Las redes de comunicación para el aprendizaje y la formación docente universitaria. EDUTEC, Revista Electrónica de Tecnología Educativa, 23, 1-11. Disponible en http://edutec.rediris.es/Revelec2/revelec23/marin_romero/marin_romero.html.

Marín, V., Vázquez, A.I., Llorente, M.C., y Cabero, J. (2012). La alfabetización digital del docente universitario en el Espacio Europeo de Educación Superior. EDUTEC, Revista Electrónica de Tecnología Educativa, 39. Recuperado el dd/mm/aa de http://edutec.rediris.es/Revelec2/Revelec39/alfabetizacion_digital_docente_universit ario_EEES.html

Martínez, F. (1994). Investigación y nuevas tecnologías de la comunicación en la enseñanza: el futuro inmediato. Pixel-Bit. Revista de medios y educación, 2, 3-17

Mason, R., y Rennjie, F. (2008). E-learning and Social Networking Handbook. Resources for Higher Education. Routledge

Means et al. (2009). Evaluation of Evidence-Based Practices in Online Learning: A Meta-Analysis and Review of Online Learning Studies. US Department of Education. [http://www2.ed.gov/rschstat/eval/tech/evidencebased-practices/finalreport.pdf]

Moran, L. y Myringer, B. (1999). Flexible learning and university change. In Harry,K. (ed.): Higher Education Through Open and Distance Learning. London: Routledge

Mott, J., y Wiley, D. (2009). Open for Learning: The CMS and the Open Learning Network. In Education, 15(2). Technology \& Social Media (Special Issue, Part 1). Retrieved from http://ineducation.ca/article/open-learning-cms-and-open-learningnetwork

Paulsen, M. F. (2004). Online Education. Public Services Review: Nordic States. 
Prendes, M.P. (2007). "Internet aplicado a la educación: estrategias didácticas y metodologías". Cabero, J. (coord.). Nuevas tecnologías aplicadas a la educación. Madrid. McGraw-Hil (pp. 205-222).

Punie, Y., Ala-Mutka, K., Redecker, C. (2008). School's Over: Learning Spaces in Europe in 2020: An Imagining Exercise on the Future of Learning. European Commission: Institute for Prospective Technological Studies, Sevilla.

Redecker, C. et al. (2009). Learning 2.0: The Impact of Web 2. Innovations on Education and Training in Europe. http://ipts.jrc.ec.europa.eu. [consulta, 14-1-2010].

Reeves, T. C. (2000). Enhancing the Worth of Instructional Technology Research through "Design Experiments" and Other Development Research Strategies. International Perspectives on Instructional Technology Research for the 21st Century Symposium. New Orleans, LA, USA.

Reeves, T. C. (2006). Design research from the technology perspective. In J. van den Akker, K. Gravemeijer, S. McKenney, \& N. Nieveen (Eds.): Educational design research (pp. 86-109). London: Routledge.

Reigeluth, C., y Frick, T. (1999). Formative research: A methology for Creating and Improving Design Theories. En Reigeluth, C. (Ed.): Instructional-Design Teheories and Models. A New Paradigm of Instructional Theory (Vol. II). Lawrence Erlbaum. Mahwah (NJ), USA. (pp. 633-652).

Rhodes, D. (1994). Sharing the vision: Creating and Communicating Common Goals, and Understanding the Nature of Change in Education. En Kearsley,G. y Linch,W. (De.): Educational Technology: Leadership perspectives (pp. 29-38). Educational Technology Pub. Englewood Clifs, NJ.

Richey, R., y Klein, J. (2007). Design and Development Research: Methods, Strategies, and Issues. NY Routledge

Rudd, T., Gifford, C., Morrison, J., y Facer, K. (2006). What if...? Re-imagining learning spaces Opening Education reports. Bristol: Futurelab.

Salinas J., y Marin,V. (2010). Una propuesta para el fortalecimiento de la investigación en nuestro campo: Panel Internacional de Investigación de Tecnología Educativa. XIII Congreso Internacional EDUTEC 2010: E-Learning 2.0: Enseñar y Aprender en la Sociedad del Conocimiento. Bilbao, España.

Salinas, J. (1995). Organización escolar y redes: Los nuevos escenarios del aprendizaje. En CABERO, J. y MARTINEZ, F. (Coord.): Nuevos canales de comunicación en la enseñanza (pp. 89-117). Centro de Estudios Ramón Areces, Madrid.

Salinas, J. (1999). Enseñanza flexible, aprendizaje abierto. Las redes como herramienta para la formación. EDUTEC, Revista Electrónica de Tecnología Educativa, 10. http://edutec.rediris.es/Revelec2/Revelec10/revelec10.html 
Salinas, J. (2004). Cambios metodológicos con las TIC. Estrategias didácticas y entornos virtuales de enseñanza-aprendizaje. Bordón, 56(3-4), 469-481.

Salinas, J. (2005a). La gestión de los Entornos Virtuales de Formación. En Seminario Internacional: La calidad de la formación en red en el Espacio Europeo de Educación Superior. Tarragona, 19-22 septiembre.

Salinas, J. (2005b). Nuevos escenarios de aprendizaje. Grupo CIFO: IV Congreso de Formación para el Trabajo (pp. 421-431). IFES, Fundación Forcem y Universidad de Vigo, 2005.

Salinas, J. (2008). Modelos didácticos en los campus virtuales universitarios: patrones metodológicos generados por los profesores en procesos de enseñanza-aprendizaje en entornos virtuales. EA2007-0121. http:/gte.uib.es/pape/gte/proyectos/modelosdidacticos-en-los-campus-virtuales-universitarios-patrones-metodologicos-generados

Salinas, J. (2009a). Hacia nuevas formas metodológicas en e-learning. Formación XXI. Revista de Formación y empleo, 12, abril 2009. http://formacionxxi.com/porqualMagazine/do/get/magazineArticle/2009/03/text/xml/ Hacia_nuevas_formas_metodologicas_en_e_learning.xml.html

Salinas, J. (2009b). Innovación educativa y TIC en el ámbito universitario: Entornos institucionales, sociales y personales de aprendizaje. En II Congreso Internacional de Educación a Distancia y TIC. Lima, Perú, 2009

Salinas, J. (2009c). Modelos emergentes en entornos virtuales de aprendizaje. En Congresso Internacional EDUTEC 2009: Sociedade do Conhecimento e Meio Ambiente: Sinergia Científica. Manaus (Br). http://gte.uib.es/pape/gte/content/modelos-emergentes-en-entornos-virtuales-deaprendizaje

Salinas, J., Marín, V., y Escandell, C. (2011). A Case of an Institutional PLE: Integrating VLEs and E-Portfolios for Students. The PLE Conference 2011. Southampton, UK. Retrieved from http://journal.webscience.org/585/

Salinas, J., Pérez, A. y de Bento, B. (2008). Metodologías centradas en el alumno para el aprendizaje en red. Síntesis: Madrid.

Salmon, G. (2004). E-Moderating . The Key to Teaching and Learning Online. Ed. Routledge Falmer (UK).

Santos, C., \& Pedro, L. (2010). What's the role for institutions in PLEs? The case of SAPO Campus. The PLE Conference 2010. Recuperado de: http://www.slideshare.net/csantos/whats-the-role-for-institutions-in-ples-the-case-ofsapo-campus

Siemens, G. (2006). Connectivism - Learning Theory or Pastime for the Self-Amused? Recuperado de: http://www.elearnspace.org/Articles/connectivism_self-amused.htm 
Souter, K., Riddle, M., Sellers, W., y Keppell, M. (2011). Spaces for Knowledge génération. Final Report. Department of Education, Employment and Workplace Relations, Sidney (Au). Recuperado de: http://www.skgproject.com

Stokes, D. E. (1997). Pasteur's quadrant: Basic science and technological innovation. Washington, DC: Brookings Institution Press.

Tait, A. (1999). The convergence of distance and conventional education. Some implications for policy. En Tait,A. Y Mills, R. (eds.): The Convergence of Distance and Conventional Education. Pattenrs of flexibility for the individual learner (pp. 141-149). Routledge, New York..

Taraghi, B., Ebner, M., y Schaffert, S. (2009). Personal Learning Environments for Higher Education: A Mashup Based Widget Concept. Proceedings of the Second Workshop on Mashup Personal Learning Environments (MUPPLE09). In 4th European Conference on Technology-Enhanced Learning (Nice, France): V. 506, 18.

The Design-Based Research Collective. (2003). Design-Based Research: An Emerging Paradigm for Educational Inquiry. Educational Researcher, 32(1), 5-8. doi:10.3102/0013189X032001005

Van den Akker, J. (1999). Principles and methods of development research. In J. van den Akker, N. Nieveen, R. M. Branch, K. L. Gustafson, \& T. Plomp, (Eds.), Design methodology and developmental research in education and training (pp. 1-14). The Netherlands: Kluwer Academic Publishers

Van den Brande, L. (1993). Flexible and Distance Learning. John Wiley \& Sons, Chicherter (UK).

Weller, M. (2007). Virtual Learning Environments: Using, Choosing and Developing Your VLE. London: Routledge.

Wenger, E., McDermott, R., y Zinder, W. (2002). Cultivating communities of practice. A guide to managing knowledge. Harvard Business School Press

Wheleer, S. (2011). Learning and teaching in the digital age. $8^{\circ}$ International Teacher Training Seminar, Barcelona.

White, S., y Davis, H. C. (2011). Rich and personal revisited: translating ambitions for an institutional personal learning environment into a reality. The PLE Conference 2011. Southampton, UK. Recuperado de: http://eprints.ecs.soton.ac.uk/22140/

Wilson, S., Sharples, P., Griffiths, D., y Popat, K. (2009). Moodle Wave: Reinventing the VLE using Widget technologies. University of Bolton, UK. Recuperado de: http://dspace.ou.nl/handle/1820/2238 
Zawacki-Richter, O. et al. (2009). Review of Distance Education Research (2000 to 2008): Analysis of Research Areas, Methods and Authorship Patterns. International Review of Research in Open and Distance Learning, 6(10), 21-49. 